\title{
L'approche de la gestion du risque radon dans les habitations aux Etats-Unis*
}

\author{
André BOUVILLE $\star \star$
}

\begin{abstract}
RÉSUMÉ
Le radon dans les habitations est un sujet dont le grand public a pris connaissance en 1985 à la suite de la découverte de concentrations élevées dans des maisons construites dans la zone géologique du Reading Prong en Pennsylvanie. Le niveau d'action recommandé par I'U.S. Environmental protection agency (EPA) est de $4 \mathrm{pCi}^{-1}\left(150 \mathrm{~Bq} \mathrm{~m}^{-3}\right)$ pour la concentration moyenne annuelle en radon gaz dans l'air des habitations. La distribution statistique des niveaux moyens de radon n'est pas encore bien établie mais il semble qu'elle soit log normale avec une moyenne géométrique de l'ordre de $1 \mathrm{pCi}{ }^{-1}\left(37 \mathrm{~Bq} \mathrm{~m}^{-3}\right)$ et un écart-type géométrique d'environ 2,5. Le nombre d'habitations dans lesquelles les concentrations en radon gaz sont supérieures à $4 \mathrm{pCi} \mathrm{I}^{-1}\left(150 \mathrm{~Bq} \mathrm{~m}^{-3}\right)$ serait par conséquent de plusieurs millions. Les très nombreuses mesures effectuées depuis 1985 ont révélé des concentrations élevées de radon dans presque tous les Etats.
\end{abstract}

EPA assure le conseil et l'assistance technique auprès des Etats. II est habituellement demandé aux particuliers d'assurer la charge financière des òpérations de réduction des concentrations de radon dans leurs habitations mais celles-ci n'ont aucun caractère obligatoire.

D'importants programmes de recherche sont consacrés à tous les aspects touchant au radon dans les habitations et aux risques sanitaires qui en découlent.

\section{ABSTRACT}

The general public heard of the problem of indoor radon in 1985 when high concentrations were discovered in dwellings built in the geological area of Reading Prong in Pennsylvania. The action level recommended by the U.S. Environmental protection agency (EPA) was $4 \mathrm{pCi}^{-1}\left(150 \mathrm{~Bq} \mathrm{~m}^{-3}\right)$ for the mean annual concentration in radon gas in indoor air. The statistical distribution of radon mean levels has not yet been well established, but it seems to be lognormal with a geometric mean of about $1 \mathrm{pCi}^{-1}\left(37 \mathrm{~Bq} \mathrm{~m}^{-3}\right)$ and a geometric standard deviation of about 2.5. The number of dwellings with radon gas concentrations above $4 \mathrm{pCi} \mathrm{I}^{-1}\left(150 \mathrm{~Bq} \mathrm{~m}^{-3}\right)$ would thus reach several millions. The very numerous measurements carried out since 1985 have demonstrated high radon levels in nearly all States.

EPA provides the States with advice and technical assistance. Individuals are usually asked to cover the mitigation expenses in their dwellings, which, however, have no compulsory character.

Important research programs are devoted to the various aspects of indoor radon and of the health risks involved.

* Communication présentée lors de la réunion SFRP "Exposition au radon dans les habitations: aspects techniques et sanitaires", Paris, 10-11 janvier 1989.

** National cancer institute, c/o Environmental measurements laboratory, 376 Hudson Street, New York, NY 10014, USA. 


\section{INTRODUCTION}

Bien que diverses études sur le radon dans les habitations aient été effectuées aux Etats-Unis depuis les années 1960, l'intérêt du grand public et des médias de ce pays n'a véritablement été suscité qu'en décembre 1984, lorsqu'un employé de la centrale nucléaire de Limerick, en Pennsylvanie, déclencha une alarme lors de son entrée dans un bâtiment. L'enquête démontra que la source de la contamination était la maison de l'employé, dans laquelle les concentrations en descendants de radon étaient de 1600 à $2000 \mathrm{pCi}^{-1}$ [4].

A la suite de cet événement spectaculaire, des programmes de mesure de radon ont été entrepris dans de nombreux Etats dans le but principal de détecter les maisons à niveau élevé et de protéger leurs habitants en abaissant ces niveaux [12]. En parallèle, un effort de recherche important, consacré à tous les aspects touchant au radon et à ses produits de filiation dans les habitations, a été mis sur pied.

L'objet de ce document est de résumer les connaissances actuelles sur les teneurs de radon dans l'air des habitations aux Etats-Unis, et de présenter la stratégie de contrôle utilisée ainsi que les principaux axes de recherche.

\section{CONCENTRATIONS EN RADON DANS LES HABITATIONS AUX ETATS-UNIS}

\section{Distribution}

A l'heure actuelle, aucune étude de grande dimension n'a été effectuée à l'échelle nationale pour déterminer la distribution de la concentration annuelle moyenne en radon de l'air des habitations. Cependant, de nombreuses campagnes de mesure, souvent biaisées en faveur des concentrations élevées et ayant généralement de courte durée d'exposition, ont été faites dans un cadre local ou régional.

L'étude qui actuellement renseigne le plus sur les niveaux annuels moyens de radon est celle de COHEN [13] qui plaça des dosimètres pendant une année dans les habitations d'environ 450 enseignants dans une centaine d'universités réparties dans l'ensemble du pays. La distribution obtenue est log normale, avec une moyenne géométrique de $38 \mathrm{~Bq} \mathrm{~m}^{-3}$ $\left(1 \mathrm{pCi} \mathrm{I}^{-1}\right)$, et un écart-type géométrique de 2,36 [3]. Cette étude est malheureusement de dimensions modestes et porte sur des habitations qui ne sont probablement pas représentatives de l'ensemble des résidences des Etats-Unis. Ces résultats sont toutefois conformes à ceux de NERO [9], qui agrégea les mesures de 22 études locales effectuées avant 1985 et les analysa de manière à obtenir des moyennes annuelles: sur cet échantillon, comportant moins d'un millier d'habitations, il obtint, lui aussi, une distribution log normale avec une moyenne géométrique de $0,9 \mathrm{pCi} \mathrm{I}^{-1}\left(33 \mathrm{~Bq} \mathrm{~m}^{-3}\right)$ et un écart-type géométrique de 2,8 [9]. 
II faut, par ailleurs, noter qu'un nombre très important de mesures a été effectué depuis 1985, mais souvent dans des conditions pessimistes et dans les habitations susceptibles de présenter des niveaux de radon élevés. Par exemple, EPA a développé un programme d'assistance aux Etats désireux de déterminer une distribution statistique du radon dans les habitations; dans ce cadre, une première série de mesures est effectuée en hiver, au sous-sol et au rez-de-chaussée, toutes ouvertures fermées et sur un court temps d'exposition, afin de détecter rapidement les zones à risque. Les résultats sont actuellement disponibles pour 14 Etats et portent sur 19000 mesures [6]: la moyenne géométrique des concentrations en radon au rezde-chaussée varie d'un Etat à l'autre de 0,6 à $2,3 \mathrm{pCi} \mathrm{I}^{-1}\left(20\right.$ à $\left.85 \mathrm{~Bq} \mathrm{~m}^{-3}\right)$ tandis que les niveaux excédant $4 \mathrm{pCi} \mathrm{I}^{-1}\left(150 \mathrm{~Bq} \mathrm{~m}^{-3}\right)$ s'étagent entre 3 et $28 \%$ du total des habitations.

Certains des résultats obtenus par des entreprises commerciales ont également été analysés:

(a) Terradex a traité 91000 de ses mesures effectuées avec des détecteurs de traces [14]. La moyenne géométrique des concentrations en radon, pondérée par la population de chaque Etat, est de $1,9 \mathrm{pCi}^{-1}\left(71 \mathrm{~Bq} \mathrm{~m}^{-3}\right)$; $20 \%$ des résultats dépasse $4 \mathrm{pCi} \mathrm{I}^{-1}\left(150 \mathrm{~Bq} \mathrm{~m}^{-3}\right)$;

(b) Key technology, Inc. a rassemblé les résultats de plus de 61000 mesures de radon effectuées à l'aide de conteneurs de charbon actif [15]. Des concentrations supérieures à $4 \mathrm{pCr} \mathrm{I}^{-1}\left(150 \mathrm{~Bq} \mathrm{~m}^{-3}\right)$ ont été trouvées dans la plupart des Etats, dans des proportions variant généralement entre 10 et $40 \%$.

Enfin, il paraît utile d'indiquer qu'EPA a décidé d'entreprendre une étude nationale sur la distribution des concentrations moyennes annuelles en radon dans l'air des habitations.

\section{Niveaux élevés}

Les niveaux de radon élevés dans les habitations ont d'abord été liés à l'utilisation de déchets industriels dans l'industrie du bâtiment. Ce fut le cas, par exemple, de constructions bâties avec ou sur des stériles de minerai d'uranium à Grand Junction, dans le Colorado, pour lesquelles des concentrations en produits de filiation du radon s'élevant jusqu'à environ $100 \mathrm{pCi} \mathrm{I}^{-1}$ $\left(4000 \mathrm{~Bq} \mathrm{~m}{ }^{-3}\right)$ ont été obtenues dans divers types de structure dont des maisons et des écoles [9]. Un autre exemple important est celui d'habitations construites sur l'emplacement d'anciennes carrières de phosphate en Floride: sur un échantillon de 133 structures, environ un tiers présentait des concentrations en produits de filiation du radon supérieures à $1 \mathrm{pCi}^{-1}$ (37 $\mathrm{Bq} \mathrm{m} \mathrm{m}^{-3}$ ) tandis que quelques-unes étaient supérieures à $5 \mathrm{pCi} \mathrm{^{-1 }}$ $\left(185 \mathrm{~Bq} \mathrm{~m}^{-3}\right.$ ). Ces résultats, obtenus au cours des années 1960 et 1970 , ont conduit à l'établissement de recommandations visant à abaisser les risques potentiels dans les maisons construites ou à construire qui seront présentées plus bas. 
Ce n'est qu'à la suite de la découverte de niveaux élevés de radon dans l'habitation de l'employé de la centrale nucléaire de Limerick, en Pennsylvanie, que d'importantes campagnes de mesure montrèrent que les concentrations "naturelles" en radon dans l'air des habitations des EtatsUnis étaient bien plus élevées que ce qui était préalablement supposé, au moins pour certaines régions du pays. En Pennsylvanie, davantage d'habitations avec des teneurs en radon très élevées ont été ainsi découvertes à proximité de la maison de l'employé de la centrale. La source de contamination, d'origine naturelle, est attribuée à une formation géologique granitique, appelée Reading Prong, qui s'étend dans une partie des Etats de Pennsylvanie, de New-York et du New-Jersey. Une campagne de mesures, effectuée dans des conditions pessimistes, dans environ 20000 maisons a révélé des concentrations en radon supérieures à $4 \mathrm{pCi} \mathrm{l}^{-1}\left(148 \mathrm{~Bq} \mathrm{~m}^{-3}\right)$ dans $60 \%$ d'entre elles, supérieures à $20 \mathrm{pCi} \mathrm{I}^{-1}\left(740 \mathrm{~Bq} \mathrm{~m}^{-3}\right)$ dans $12 \%$ des cas, et supérieures à $200 \mathrm{pCi} \mathrm{I}^{-1}\left(7400 \mathrm{~Bq} \mathrm{~m}^{-3}\right)$ dans $0,5 \%$ des habitations [4]. Un programme similaire dans l'Etat du New-Jersey a conduit à la découverte d'un noyau de maisons à niveau de radon élevé à Clinton: dans 105 maisons, les concentrations en radon dans les sous-sols sont toutes supérieu-

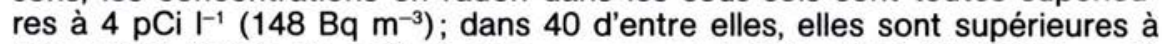
$200 \mathrm{pCi}^{-1}\left(7400 \mathrm{~Bq} \mathrm{~m}^{-3}\right)$ et elles surpassent $1000 \mathrm{pCi}^{-1}\left(37000 \mathrm{~Bq} \mathrm{~m}^{-3}\right)$ dans 5 maisons. Clinton est située à proximité du Reading Prong mais se trouve, en fait, dans une zone dolomitique, ce qui montre qu'une variété de conditions géologiques peut donner naissance à des niveaux élevés de radon.

II est vraisemblable que les campagnes de mesure entreprises dans d'autres Etats conduiront, elles aussi, à la découverte de situations analogues.

\section{STRATÉGIE DE CONTRÔLE}

\section{Réglementation}

Deux types de situation doivent être clairement distingués:

(a) Contrôle du radon dans les bâtiments dont les concentrations élevées découlent d'activités industrielles,

(b) Contrôle du radon dans les habitations où les hautes teneurs sont d'origine naturelle.

\subsection{Contamination industrielle}

Plusieurs cas de contamination industrielle ont été considérés aux Etats-Unis:

- résidus de traitement des minerais d'uranium: selon les recommandations du "Chirurgien général", émises en 1972, des mesures d'intervention dans les résidences de Grand Junction, $\mathrm{CO}$, étaient indiquées pour des concentrations en produits de filiation du radon supérieures à $5 \mathrm{pCi}^{1-1}$ (185 $\mathrm{Bq} \mathrm{m} \mathrm{m}^{-3}$ ) et suggérées au-dessus de $1 \mathrm{pCi}^{-1}\left(37 \mathrm{~Bq} \mathrm{~m}^{-3}\right)$ [10]; 
- résidus de l'industrie du phosphate: I'EPA a recommandé, en 1979, qu'une intervention soit faite dans les résidences de Floride dans lesquelles les concentrations en produits de filiation du radon étaient supérieures à $2 \mathrm{pCi} \mathrm{l}^{-1}\left(74 \mathrm{~Bq} \mathrm{~m}^{-3}\right)$. EPA a également recommandé que des mesures d'intervention soient étudiées, structure par structure, pour des niveaux plus bas mais a noté que celles-ci ne seraient probablement pas justifiées pour des concentrations n'excédant pas $0,5 \mathrm{pCi}^{-1}\left(18 \mathrm{~Bq} \mathrm{~m}^{-3}\right)$ en descendants du radon au-dessus du bruit de fond normal à l'intérieur des habitations [9] ;

- résidus de l'industrie du radium: de nombreuses entreprises qui utilisaient au cours de la première moitié du siècle du radium à des fins militaires ou commerciales ont laissé derrière elles des résidus. Dans le New-Jersey, par exemple, des concentrations en radon élevées ont été observées dans plusieurs communautés [9]. EPA et le Center for disease control ont recommandé que des mesures soient prises dans les maisons ayant une concentration en produits de filiation du radon supérieure à $2 \mathrm{pCi}$ $\mathrm{I}^{-1}\left(74 \mathrm{~Bq} \mathrm{~m}^{-3}\right)$.

\subsection{Présence d'origine naturelle}

En 1986, EPA a recommandé que les concentrations moyennes annuelles en radon dans l'air des habitations soient abaissées ou maintenues au-dessous de $4 \mathrm{pCil}^{-1}\left(150 \mathrm{~Bq} \mathrm{~m}^{-3}\right)$ ou au-dessous de $2 \mathrm{pCi}^{-1}\left(74 \mathrm{~Bq} \mathrm{~m}^{-3}\right)$ au cas où ce sont les produits de filiation du radon qui sont mesurés. EPA est donc cohérent avec lui-même en recommandant le même niveau d'action en cas de contamination industrielle et en cas d'origine naturelle.

La marche à suivre recommandée par EPA est la suivante:

(a) Mesure préliminaire effectuée dans des conditions pessimistes et sur un court temps d'exposition (mesure dans le sous-sol si possible, sinon au rez-de-chaussée; portes et fenêtres maintenues fermées $12 \mathrm{~h}$ au moins avant le début de l'exposition et pendant toute la durée de celle-ci);

(b) Mesure de confirmation si la mesure préliminaire conduit à une concentration en radon supérieure à $4 \mathrm{pCi} \mathrm{I}^{-1}\left(150 \mathrm{~Bq} \mathrm{~m}^{-3}\right)$; cette mesure de confirmation, toujours effectuée dans des conditions pessimistes, porte sur une période d'exposition d'autant plus longue que le niveau de radon est plus faible;

(c) Intervention s'il est confirmé que la concentration en radon est supérieure à $4 \mathrm{pCi}^{-1}\left(150 \mathrm{~Bq} \mathrm{~m}^{-3}\right)$; la rapidité avec laquelle l'intervention est faite dépend de la concentration en radon:

- quelques semaines pour des concentrations de plus de $200 \mathrm{pCi}^{-1}$ (7 $400 \mathrm{~Bq} \mathrm{~m} \mathrm{~m}^{-3}$ );

- quelques mois pour des concentrations comprises entre 20 et

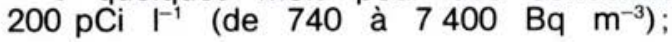

- plusieurs années pour des concentrations comprises entre 4 et $20 \mathrm{pCi}^{-1} \cdot\left(\right.$ de 150 à $740 \mathrm{~Bq} \mathrm{m^{-3 }}$ ).

II faut bien remarquer que tout cela n'est que recommandation et que le particulier lui-même doit décider s'il veut connaître la concentration en 
radon dans l'air de son habitation (mesure généralement faite à ses frais) et s'il veut prendre des mesures pour abaisser cette concentration (également à ses frais dans la plupart des cas).

En septembre 1988, EPA a de nouveau invité "virtuellement tout le monde aux Etats-Unis" à faire établir la concentration en radon dans l'air de son habitation et à faire procéder à des mesures de réduction, si nécessaire [1].

\section{Mise en cuvre}

Ici aussi, il convient de séparer les cas de contamination industrielle de ceux où la présence du radon est d'origine naturelle.

\subsection{Concentration industrielle}

Les opérations de réduction des niveaux de radon ("mitigation") sont prises en charge par les autorités:

(1) Grand Junction, CO : environ 3000 maisons furent classées comme étant contaminées. Le programme d'intervention, qui nécessite des travaux majeurs d'excavation et de reconstruction, revient à plus de 30000 dollars US par maison [8].

(2) Floride: le programme d'action est surtout axé sur la llmitation des niveaux de radon dans les maisons à construire plutôt que sur la "mitigation" dans les maisons existantes. Après consultation avec EPA, l'Etat de la Floride a promulgué en avril 1986 une réglementation sur les pratiques de construction. Cette réglementation est applicable uniquement pour certaines zones dans lesquelles l'exploitation de phosphates a pris place auparavant, mais il est prévu qu'elle puisse également s'appliquer à d'autres régions à niveau de radon élevé qui seraient définies par l'Etat. Cependant, peu après la promulgation de la loi, des difficultés apparurent. La carte définissant les zones d'application de la nouvelle réglementation s'avéra sujette à controverse. L'Etat décida de suspendre l'application de la réglementation tant qu'une étude sur les niveaux de radon dans tout l'Etat de la Floride ne serait pas terminée et que les zones géographiques d'application de la loi ne seraient pas réexaminées. Le dossier est en attente [4].

(3) New-Jersey : l'Etat du New-Jersey, en association avec les autorités fédérales, a remédié à la situation en retirant le sol contaminé par le radium. L'intervention a, cependant, suscité un autre problème, qui est celui du devenir de ces déchets radioactifs [9].

\subsection{Présence d'origine naturelle}

Deux niveaux administratifs sont à considérer:

2.2.1. Niveau fédéral : EPA joue auprès des Etats un rôle de conseil et prête assistance technique. Dès que l'importance de la situation liée au Reading Prong fut connue en 1985, EPA entreprit une série d'actions (appelée "Radon action program" afin d'aider la Pennsylvanie et 
les autres Etats à faire face au problème posé par les concentrations élevées de radon dans l'air des habitations. Ce programme inclut:

(a) L'établissement de protocoles de mesure de radon et de ses produits de filiation;

(b) La possibilité d'installations de référence, grâce auxquelles les laboratoires gouvernementaux et commerciaux peuvent faire la preuve de leur maîtrise des techniques de mesure du radon et de ses descendants;

(c) La démonstration de techniques de "mitigation", notamment effectuée en Pennsylvanie et dans le New-Jersey;

(d) La publication de plusieurs brochures destinées au grand public ou aux professionnels [13-17], lesquelles ont trait aux sources de radon dans les maisons, aux niveaux recommandés, ainsi qu'aux techniques de mesure et de réduction des niveaux;

(e) L'assistance technique, aux Etats qui en font la demande, pour identifier les zones à risque potentiel, établir les causes de niveaux de radon élevés, et conseiller sur la manière d'abaisser ces niveaux.

\subsubsection{Niveau des Etats:}

Les Etats, qui ont la responsabilité du bien-être de leurs administrés, organisent et/ou supervisent de leur mieux les actions menées dans le domaine du radon. L'intérêt manifesté par le public, l'importance du programme de mesure, la manière dont les mesures d'intervention sont financées et réglementées varient d'un Etat à l'autre. Les actions entreprises par les Etats de Pennsylvanie et du New-Jersey, qui figurent parmi les plus actifs, sont ici présentées à titre d'exemple:

\section{Pennsylvanie:}

L'Etat tenta d'abord directement auprès de la population résidant sur le Reading Prong de leur laisser faire des mesures de radon dans leurs habitations. Environ $70 \%$ des personnes contactées refusèrent, préférant ne pas connaître le risque auquel elles étaient exposées. Cependant, les mesures effectuées chez les $30 \%$ des personnes qui acceptèrent permirent de révéler l'importance du problème. L'Etat prit alors les mesures suivantes:

(a) Distribution gratuite de dosimètres radon, sur demande, aux 70000 familles résidant sur le Reading Prong; environ 20000 familles acceptèrent cette offre;

(b) Installation d'un groupe de 21 techniciens dans une ville du Reading Prong avec mission de conseil et d'assistance technique;

(c) Etablissement d'un crédit de 1 million de dollars destiné essentiellement à faire la démonstration de l'efficacité des mesures de "mitigation" dans au moins 100 maisons; ce programme, mené en coopération avec EPA, fut accompli de manière satisfaisante en 1987-1988; 
(d) Réglementation de la certification des firmes commerciales désirant effectuer des mesures de radon ou des opérations de "mitigation";

(e) Etablissement d'un programme de prêts à faible taux d'intérêt destiné à aider les personnes désireuses de faire procéder à des mesures de "mitigation" dans leurs habitations.

Au mois d'août 1987, 272 opérations de "mitigation", financées par des fonds publics, étaient terminées ou en cours tandis que plus de 280 opérations similaires étaient, ou avaient été, effectuées par des entreprises commerciales [12].

\section{New-Jersey:}

L'Etat du New-Jersey, également situé sur le Reading Prong, découvrit rapidement que le nombre de maisons à risque potentiel était très élevé sur son territoire (l'estimation actuelle est de 1,6 million). Ne pouvant pas remédier au problème avec les seuls fonds publics, il se tourna vers les habitants et le secteur privé, tout en instaurant un système de garde-fous. Le programme mis sur pied, qui dispose d'un budget de 3,2 millions de dollars, consiste en:

(a) Une campagne de mesure de 6000 maisons réparties sur tout le territoire pour délimiter les zones et les types de maison à haut risque;

(b) Une intensive campagne d'information: appels téléphoniques gratuits sur une ligne spéciale ( 75000 appels avaient été reçus en octobre 1988) et distribution de plus de 40000 brochures;

(c) La création d'une licence obligatoire pour les firmes commerciales désireuses de mesurer le radon ou de se livrer à des opérations de "mitigation" ;

(d) L'offre de mesure gratuite de vérification des niveaux de radon dans

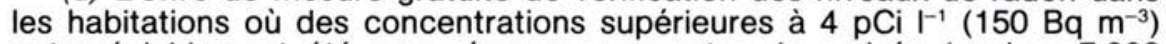
ont préalablement été mesurées par une entreprise privée (environ 7000 réponses positives);

(e) Une étude épidémiologique visant à déterminer la relation entre l'exposition au radon et le cancer du poumon.

En octobre 1988, des mesures de radon avaient été faites dans environ 200000 habitations tandis que des mesures de "mitigation" destinées à abaisser le niveau de radon au-dessous de $4 \mathrm{pCi} \mathrm{I}^{-1}\left(150 \mathrm{~Bq} \mathrm{~m}^{-3}\right)$ avaient été prises dans au moins 1500 résidences. Le suivi des mesures dans 716 de ces habitations par un organisme d'Etat montre, cependant, que les niveaux après "mitigation" excèdent $4 \mathrm{pCi}^{-1}\left(150 \mathrm{~Bq} \mathrm{~m}^{-3}\right)$ dans $64 \%$ des maisons et $20 \mathrm{pCi}^{-1}\left(740 \mathrm{~Bq} \mathrm{~m}^{-3}\right)$ dans $13 \%$ des cas [5]. Le coût moyen par maison, déterminé sur la base des rapports fournis par les entreprises commerciales, est de 1300 dollars avec une gamme de 200 à 8600 dollars. 


\section{PROGRAMMES DE RECHERCHE}

Des programmes de recherche importants ont été mis en place dans tous les domaines touchant au radon dans les habitations. Bien que certains Etats possèdent leurs programmes propres, ceux-ci sont, le plus souvent, exécutés en liaison avec les agences fédérales qui semblent financer la grande majorité des études. Au niveau fédéral, les programmes sont coordonnés de manière informelle par le "Radon work group" du "Committee on indoor air quality". Le Department of energy joue un rôle de leader dans les études de recherche fondamentale tandis qu'EPA prend de plus en plus d'importance dans les études appliquées. Une présentation détaillée des programmes de recherche n'a pas sa place dans ce domaine, mais il paraît utile d'en indiquer les grands axes:

(a) Abondance et transferts du radon dans le sol: identification des zones à sol poreux et à concentrations en uranium élevées sur la base de données pédologiques, géologiques et radiométriques;

(b) Modes d'entrée du radon dans les maisons: étude des corrélations entre le flux de radon entrant dans les maisons et la concentration en radon dans le sol, la différence de pression de part et d'autre des fondations, la température à l'intérieur et à l'extérieur des maisons, la direction et la force du vent, le degré d'humidité du sol,...

(c) "Mitigation" du radon: mise au point de techniques nouvelles, amélioration des techniques existantes et détermination de leur efficacité à long terme;

(d) Dosimétrie: identification de la fraction libre des descendants du radon, détermination du type et de l'emplacement des cellules responsables du cancer du poumon;

(e) Effets sanitaires: études épidémiologiques, expérimentation animale.

\section{CONCLUSION}

Le radon dans les habitations est un sujet dont le grand public a pris connaissance en 1985 à la suite de la découverte de concentrations élevées dans des maisons construites dans la zone géologique du Reading Prong en Pennsylvanie. Le niveau d'action recommandé par EPA est de $4 \mathrm{pCi}^{-1}$ $\left(150 \mathrm{~Bq} \mathrm{~m} \mathrm{~m}^{-3}\right.$ ) pour la concentration moyenne annuelle en radon gaz dans l'air des habitations. Ce niveau d'action est analogue à ceux qui avaient auparavant été adoptés dans des cas de contamination dus à des activités industrielles.

La distribution statistique des niveaux moyens de radon n'est pas encore bien établie, mais il semble qu'elle soit log normale avec une moyenne géométrique de l'ordre de $1 \mathrm{pCi} \mathrm{I}^{-1}\left(37 \mathrm{~Bq} \mathrm{~m}^{-3}\right)$ et un écart-type géométrique d'environ 2,5. Les très nombreuses mesures effectuées depuis 1985 ont révélé des concentrations élevées de radon dans presque tous les Etats. 
EPA assure le conseil et l'assistance technique auprès des Etats. II est habituellement demandé aux participants d'assurer la charge financière des opérations de réduction des concentrations de radon dans leurs habitations, mais celles-ci n'ont aucun caractère obligatoire. Du fait que les particuliers doivent supporter les frais des opérations de "mitigation", ils ne montrent qu'un enthousiasme modéré à se livrer à de telles opérations, malgré la pression des autorités de santé publique. Cependant, le nombre absolu d'habitations dans lesquelles des mesures de radon ont été faites et des opérations de "mitigation" effectuées est impressionnant.

Par ailleurs, d'importants programmes de recherche sont consacrés à tous les aspects touchant au radon dans les habitations et aux risques sanitaires qui en découlent.

\section{RÉFÉRENCES}

[1] EPA urges all US citizens to test homes for radon gas. Air Water Pollut. Report, 19 Sept. 1988, p. 322.

[2] ALTER H.W., OSWALD R.A. - Nationwide distribution of indoor radon measurements. The 1988 Symposium on radon and radon reduction technology, Denver, 17-21 October 1988.

[3] COHEN B.L. - A national survey of radon-222 in US homes and correlating factors. Health Phys., 1986, 51, 175-183.

[4] COTHERN C.R. et SMITH J.E., Jr (Eds.) - Environmental radon, New-York: Plenum Press, 1987.

[5] De PIERRO N. et CAHILL M. - Radon reduction efforts in New Jersey. The 1988 Symposium on radon and radon reduction technology, Denver, 17-21 October 1988.

[6] DZIUBAN J.A., WHITE S.B., BERGSTEN J.W., ALEXANDER B.V. - Residential radon survey of fourteen states. The 1988 Symposium on radon and radon reduction technology, Denver, 17-21 October 1988.

[7] NATIONAL COUNCIL ON RADIATION PROTECTION AND MEASUREMENTS (NCRP) - Evaluation of occupational and environmental exposures to radon and radon daughters in the United States (NCRP report, 78). Bethesda: NCRP, 1984.

[8] NATIONAL COUNCIL ON RADIATION PROTECTION AND MEASUREMENTS (NCRP) - Exposure of the population in the United State and Canada from natural background radiation (NCRP report, 94). Bethesda: NCRP, 1987.

[9] NAZAROFF W.W., NERO A.V., Jr. (Eds) - Radon and its decay products in indoor air. New-York: J. Wiley, 1988.

[10] US ATOMIC ENERGY COMMISSION (AEC) - Grand Junction remedial action: proposed criteria. Fed. Reg. 1972, 37, 203.

[11] US DEPARTMENT OF ENERGY - Radon research program, FY-1987. Rapport DOE/EV/01013 - H1, 1987.

[12] US ENVIRONMENTAL PROTECTION AGENCY (EPA) - Summary of State radon programs. Rapport EPA 520/1-87-19-1, 1987. 
[13] US ENVIRONMENTAL PROTECTION AGENCY (EPA) - A citizen's guide to radon - What is it and what to do about it. Rapport EPA-86-004, 1986.

[14] US ENVIRONMENTAL PROTECTION AGENCY (EPA) - Radon reduction methods - A homeowner's guide (2. ed.). Rapport EPA-87-010, 1987.

[15] US ENVIRONMENTAL PROTECTION AGENCY (EPA) - Removal of radon from household water. Rapport EPA-87-011, 1987.

[16] US ENVIRONMENTAL PROTECTION AGENCY (EPA) - Radon reduction in new constructions - an interim guide. Rapport EPA-87-009, 1987.

[17] US ENVIRONMENTAL PROTECTION AGENCY (EPA) - Radon reduction techniques for detached houses - technical guidance (2. ed.). Rapport EPA/625/ 5-87/019, 1988.

[18] WIBLIN C., GARLAND R. - A comparison of nationwide radon measurements by home dwellers and by real estate firms. EPA, The 1988 Symposium on Radon and radon reduction technology, Denver, 17-21 October 1988. 